\title{
Review Article \\ Microwave Sensors Based on Symmetry Properties of Resonator-Loaded Transmission Lines
}

\author{
Jordi Naqui and Ferran Martín \\ GEMMA/CIMITEC, Departament d'Enginyeria Electrònica, Universitat Autònoma de Barcelona, Bellaterra, 08193 Barcelona, Spain
}

Correspondence should be addressed to Jordi Naqui; jordi.naqui@uab.cat

Received 4 November 2014; Accepted 15 January 2015

Academic Editor: Yanbiao Liao

Copyright (C) 2015 J. Naqui and F. Martín. This is an open access article distributed under the Creative Commons Attribution License, which permits unrestricted use, distribution, and reproduction in any medium, provided the original work is properly cited.

\begin{abstract}
This review paper is focused on the design of microwave sensors using symmetry properties of transmission lines loaded with symmetric resonators. The operating principle of these sensors is presented and then several prototype devices are reported, including linear and angular displacement sensors and rotation speed sensors. The main advantage of the proposed sensors is the robustness against changing environmental conditions.
\end{abstract}

\section{Introduction}

The microwave sensors based on symmetry properties presented in this review paper are inspired by resonant-type metamaterial transmission lines [1-8], a subclass of metamaterial transmission lines (see the books [9-13] for an in-depth study of these artificial lines), where a host line is loaded with electrically small resonators and, habitually, with additional lumped or semilumped reactive components (inductors and/or capacitors). Figure 1 depicts the first reported resonant-type metamaterial transmission line [1], implemented by means of a coplanar waveguide (CPW) loaded with split ring resonators (SRRs) [14] and shunt connected inductive strips. If the fundamental resonance frequency of the SRRs is smaller than the cut-off frequency of the high pass filter that results by loading the CPW only with the inductive strips, then the SRR/strip-loaded CPW exhibits a band pass response, and wave propagation in the first allowed band is left handed (LH), or backward [1]. Backward wave propagation means that the phase and group velocities are antiparallel; namely, the wave fronts propagate to the direction opposite to energy flow, that is, towards the source. This unconventional type of propagation, due to the combined effect of the SRRs and inductive strips, is caused by the simultaneously negative effective permeability and permittivity in that band [15] and is related to the presence of SRRs and strips, respectively [1]. Indeed the SRR/strip-loaded
$\mathrm{CPW}$ is the planar counterpart of the one-dimensional bulk LH metamaterial reported in [16], where an array of SRRs is combined with metallic posts (the incident radiation must be polarized with the magnetic field axial to the SRRs and the electric field parallel to the metallic posts). In both media, the simultaneous negative sign of the effective permeability and permittivity does not preclude wave propagation, as follows from inspection of Maxwell's equations; however, wave propagation is not forward, but backward.

If the strips are removed, the pass band switches to a stop band, which can be interpreted as being due to the negative effective permeability related to the presence of the SRRs (see Figure 2). In the vicinity of the SRR fundamental resonance, the SRRs are excited by the magnetic field generated by the line. Above that frequency, the structure exhibits a negative effective permeability in a narrow band, and, below that frequency, the effective permeability is highly positive, resulting in a stop band. However, the stop band behavior of a CPW loaded only with pairs of SRRs can also be interpreted from circuit theory. Namely, the SRRs are resonators magnetically coupled to the host line, and the injected power is reflected back to the source if the signal frequency is in the vicinity of SRR resonance.

Microwave sensors based on the variation of the resonance frequency, phase, or quality factor of resonator-loaded lines have been reported [17-23]. These resonator parameters may be influenced by environmental changes (temperature, 


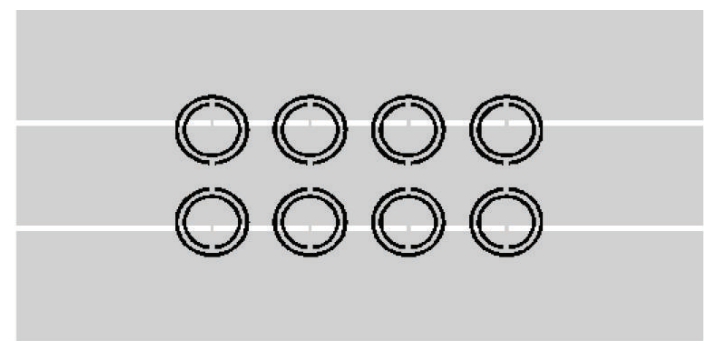

(a)

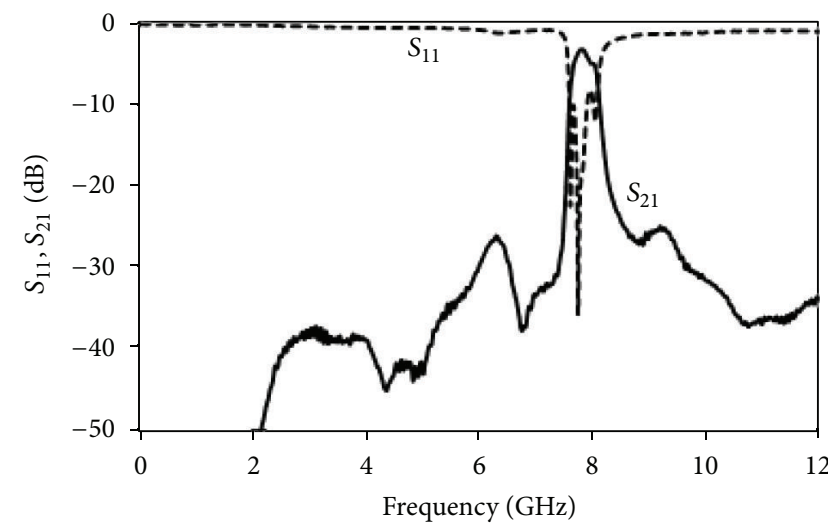

(b)

FIGURE 1: Topology of the first reported metamaterial transmission line based on a CPW loaded with pairs of shunt strips and SRRs (a) and frequency response (b), where the band pass functionality can be appreciated.

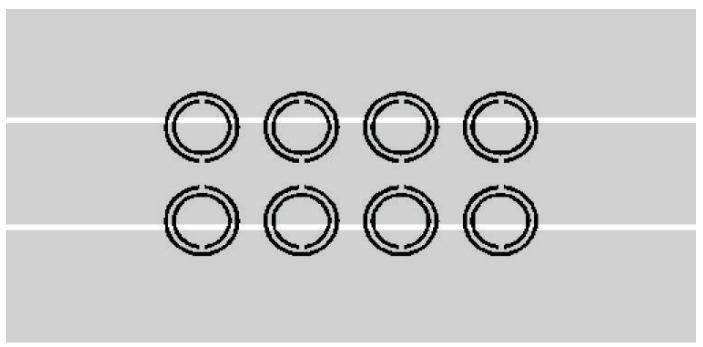

(a)

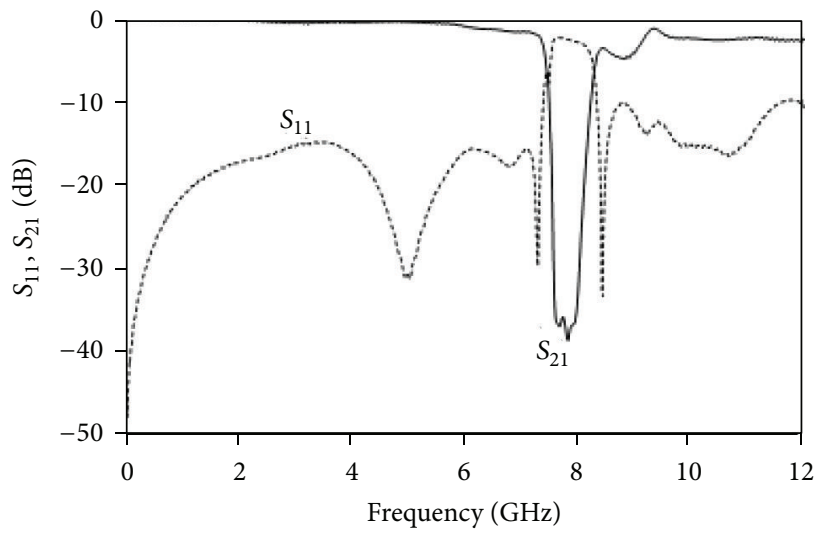

(b)

FIGURE 2: Topology of the first reported metamaterial transmission line based on a CPW loaded with pairs of SRRs (a) and frequency response (b), where the stop band functionality can be appreciated.

moisture, etc.) [24], and hence proper calibration is required, in general, before sensing (exceptions may be sensors based on differential measurements). Otherwise, measurements are likely to be affected by random errors as a result of changing ambient factors. Alternatively, a novel sensing principle, where the symmetry properties of transmission lines (host line) loaded with symmetric resonators are exploited, has been pointed out recently [25]. The resulting sensors are robust against variations of environmental conditions and especially suitable for alignment purposes since alignment is a geometrical property invariant to these conditions. Prototypes of linear and angular displacement sensors [25$34]$ as well as angular velocity sensors [33, 34] have been demonstrated. The purpose of this paper is to describe the operation principle of these sensors and to report some examples. It is also worth mentioning that some immunity to variable ambient conditions can also be attained using pairs of identical resonators (i.e., differential measurement). On the basis of this approach (beyond the scope of this paper), displacement sensors [35] and sensors for dielectric characterization [36] have been proposed by means of symmetry rupture. Other microwave and metamaterial based sensors can be found in [37-39].

\section{Sensing Principle}

The CPW structure of Figure 2 is loaded with pairs of SRRs. Regardless of the orientation of the SRRs, the magnetic field generated by the line is able to drive each resonant element of the pair, resulting in a stop band in the vicinity of the SRR fundamental resonance. Let us now consider that the CPW is loaded with a single SRR oriented with its symmetry plane aligned with the symmetry plane of the line (Figure 3(a)). Under these conditions, there is not a net axial magnetic field able to excite the particle, and the structure is transparent. The coupling between the transmission line and the resonator is therefore effectively canceled. However, if symmetry is 


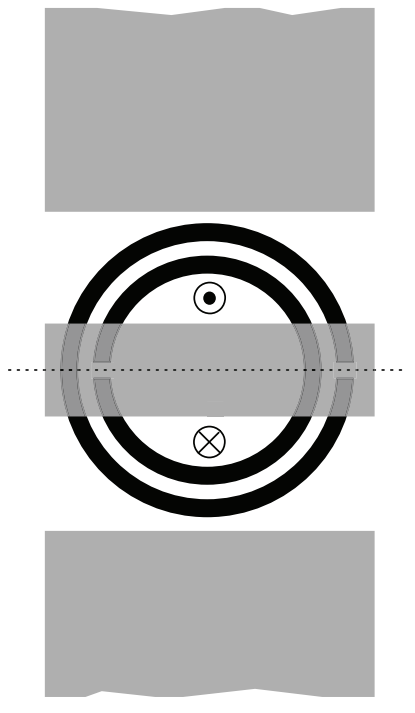

(a)

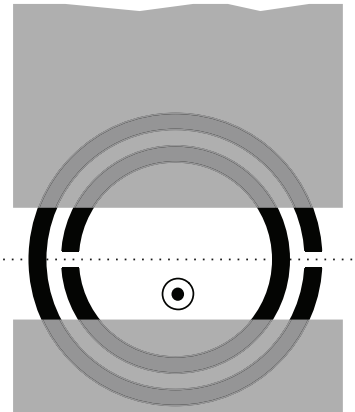

$\otimes$

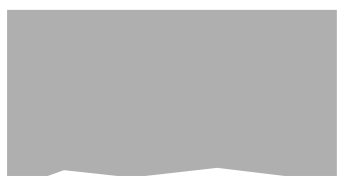

(b)

FIGURE 3: CPW loaded with a single SRR: (a) aligned symmetry planes; (b) misaligned symmetry planes. The cross and dot stand for the direction of the magnetic field, which is generated by the CPW, parallel to the SRR axis.

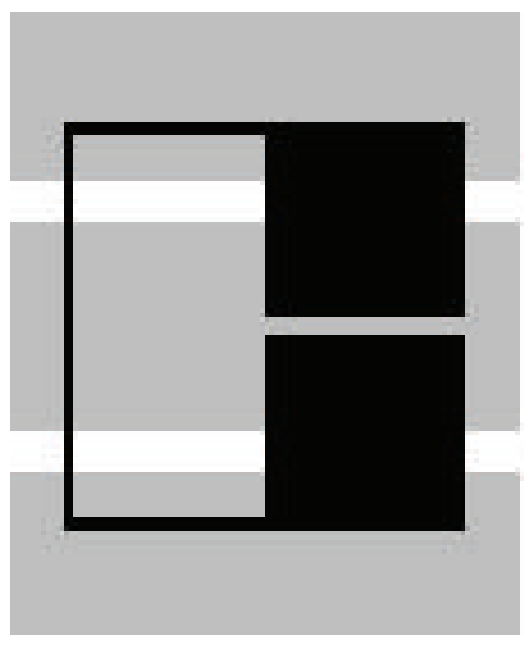

(a)

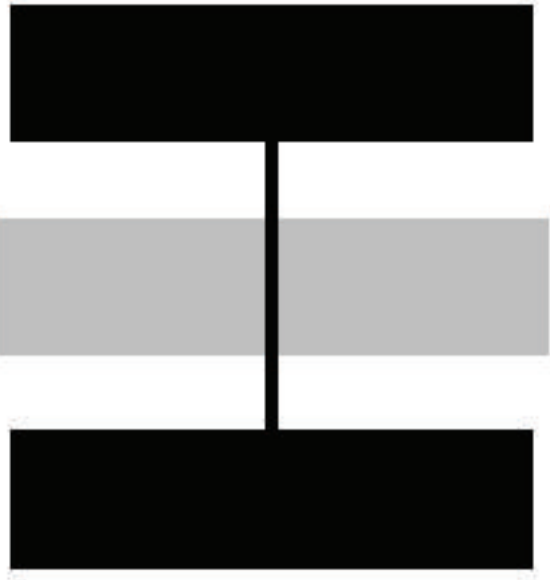

(b)

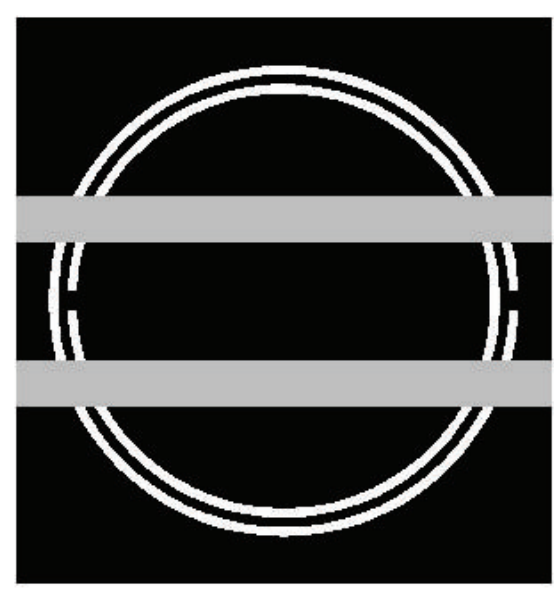

(c)

FIGURE 4: Resonator-loaded transmission lines useful for the implementation of microwave sensors based on symmetry disruption. (a) Folded SIR-loaded CPW [26]; (b) SIR-loaded microstrip line [29]; (c) CSRR-loaded differential microstrip line. In all the cases, the symmetric configuration that prevents from resonance is shown. In (b), three metal layers are required (the ground plane is not depicted). 
broken (Figure 3(b)), such perfect cancelation of magnetic field lines no longer holds; the particle is coupled to the line, and it is excited causing a notch in the transmission coefficient. The magnitude of the notch is dependent on the net coupling and hence on the level of asymmetry. Accordingly, the notch magnitude may be used for sensing purposes. Note that, with this sensing strategy, calibration for alignment measurements is not needed, since symmetry is not affected by environmental conditions. On the other hand, several types of variables, able to produce symmetry disruption, may be sensed, including linear and angular displacement, velocity, or variations in the composition (and hence dielectric permittivity) of a sample loaded to the structure.

Despite the fact that the operating principle of the proposed sensors has been explained with regard to a specific host line (CPW) and resonant element (SRR), the key aspect is to achieve transparency (total transmission) when the considered structure (host line plus resonator) is perfectly symmetric and resonance (i.e., a transmission zero) when symmetry is disrupted. A sufficient condition to achieve transmission line like behavior if symmetry is satisfied is that the symmetry planes of the line and resonator are of different electromagnetic nature (one of them a magnetic wall and the other one an electric wall). Note that this is the case of the CPW and SRR; that is, the CPW exhibits a magnetic wall at its symmetry plane for the fundamental even (CPW) mode, whereas the SRR exhibits an electric wall at its symmetry plane at the fundamental resonance. There are other combinations of transmission lines and resonators satisfying the above requirement, including CPWs and microstrip lines loaded with stepped impedance resonators (SIR) [40], or CPWs loaded with bisymmetric resonators, such as the electric-LC resonator (exhibiting an electric and a magnetic wall simultaneously in orthogonal symmetry planes) [41], or differential microstrip lines loaded with complementary split ring resonators (CSRRs) [2]. The SIR, like the SRR, exhibits an electric wall at the fundamental resonance, whereas the CSRR exhibits a magnetic wall at its symmetry plane. Some illustrative different combinations of transmission lines and resonators are depicted in Figure 4.

\section{Examples}

In this section, two different examples of microwave sensors based on symmetry properties are included: (i) a twodimensional position sensor [27] (ii) and an angular velocity sensor [33].

3.1. Two-Dimensional Position Sensor. The two-dimensional (2D) position sensor reported in this subsection is based on a right angle bended CPW loaded with a pair of SRRs in each transmission line section. For the implementation of a two-dimensional position sensor, able to measure the relative displacement between two surfaces, the SRRs must be etched on a different (movable) substrate. However, here we report a proof-of-concept where the SRRs are all etched in the back substrate side of the CPW transmission line. Therefore, we

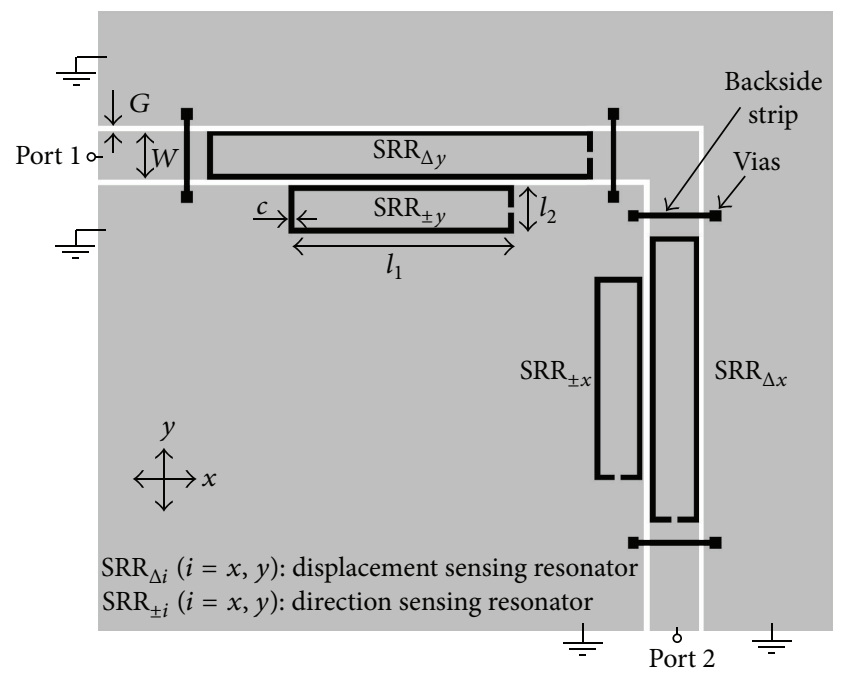

FIGURE 5: Layout of the proposed sensing device for the aligned position (i.e., the CPW and the displacement sensing SRRs are aligned). The CPW strip and slot widths are $W=1.67 \mathrm{~mm}$ and $G=$ $0.2 \mathrm{~mm}$, respectively. The dimensions of the SRRs are $l_{1}\left(\mathrm{SRR}_{\Delta x}\right)=$ $9.95 \mathrm{~mm}, l_{1}\left(\mathrm{SRR}_{ \pm x}\right)=7.05 \mathrm{~mm}, l_{1}\left(\mathrm{SRR}_{\Delta y}\right)=13.4 \mathrm{~mm}, l_{1}\left(\mathrm{SRR}_{ \pm y}\right)=$ $7.8 \mathrm{~mm}, l_{2}=1.67 \mathrm{~mm}$, and $c=0.2 \mathrm{~mm}$. The considered substrate is Rogers RO3010 with dielectric constant $\varepsilon_{r}=10.2$, thickness $h=$ $127 \mu \mathrm{m}$, and loss tangent $\tan \delta=0.0023$. From [27], reprinted with permission.

have fabricated several samples where the relative position of the SRRs with regard to the CPW transmission line has been modified. The layout of the designed sensor is depicted in Figure 5. As discussed in [25], narrow substrates are necessary to boost the sensitivity. The vias and backside strips are used to connect the ground plane regions and thus prevent the appearance of the CPW parasitic slot mode.

For a better comprehension of the principle of operation of the proposed sensor, let us consider the four different displacements indicated in Figure 6 from the aligned structure, that is, right, left, up, and down displacements. The resonance frequencies of the four SRRs are denoted as $f_{\Delta y}$, $f_{\Delta x}, f_{ \pm y}$, and $f_{ \pm x}$ (see Figure 5). It can be seen that both direction and magnitude of the displacements in the $\pm x$ - and $\pm y$-direction can be detected (by means of the resonators $\mathrm{SRR}_{ \pm x}$ and $\mathrm{SRR}_{ \pm y}$ ) and measured (by the resonators $\mathrm{SRR}_{\Delta x}$ and $\mathrm{SRR}_{\Delta y}$ ), respectively. Namely, the resonators $\mathrm{SRR}_{ \pm x, \pm y}$ are only excited if their position is shifted towards the CPW central strip, and hence it is possible to distinguish between up and down displacement, or between left and right. Indeed, such resonators act as a flag that determines the direction of motion, but not the magnitude. The magnitude of relative displacement is given by the resonators designated as $\mathrm{SRR}_{\Delta x, \Delta y}$, since these resonators are symmetrically loaded to the CPW in the unperturbed state (i.e., the one corresponding to Figure 5). Any other linear displacement is a combination of the previous ones, and hence it can also be detected and measured. As an illustration, Figure 7 shows the obtained transmission coefficient for $\Delta x=0.3 \mathrm{~mm}$ and $\Delta y=0.25 \mathrm{~mm}$. 


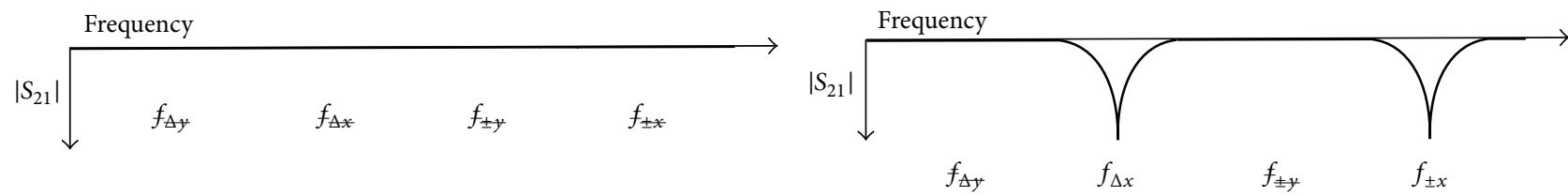

(a) $\Delta x=\Delta y=0$

(b) $+\Delta x[\Delta y=0]$

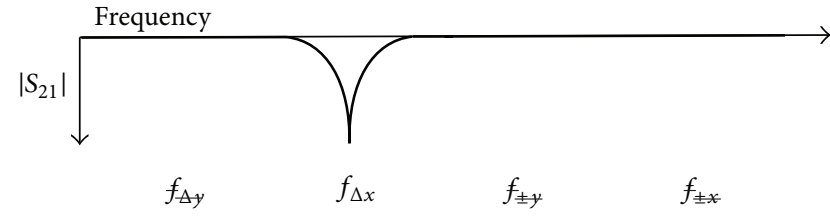

(c) $-\Delta x[\Delta y=0]$

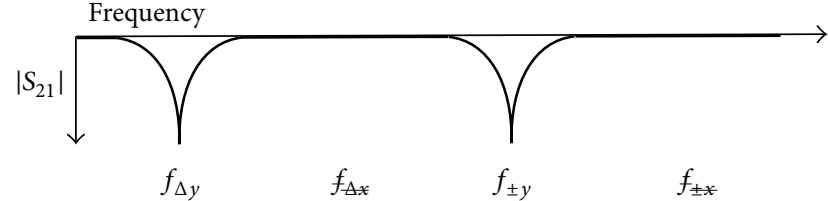

(d) $+\Delta y[\Delta x=0]$

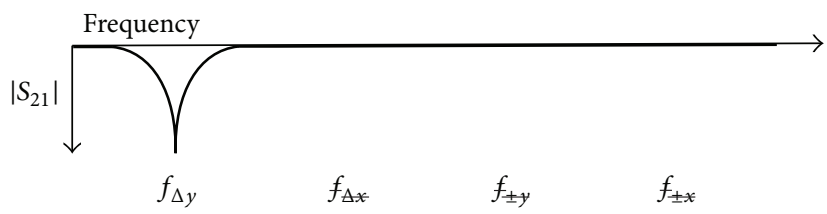

(e) $-\Delta y[\Delta x=0]$

FIGURE 6: Scheme indicating the primitive shifting operations and the resulting transmission coefficient $S_{21}$. A notch is indicative of an SRR excitation. A linear displacement in the $x$-and $y$-orientation is indicated as $\Delta x$ and $\Delta y$, respectively, relative to the aligned position (i.e., $\Delta x$ $=\Delta y=0)$. From [27], reprinted with permission.

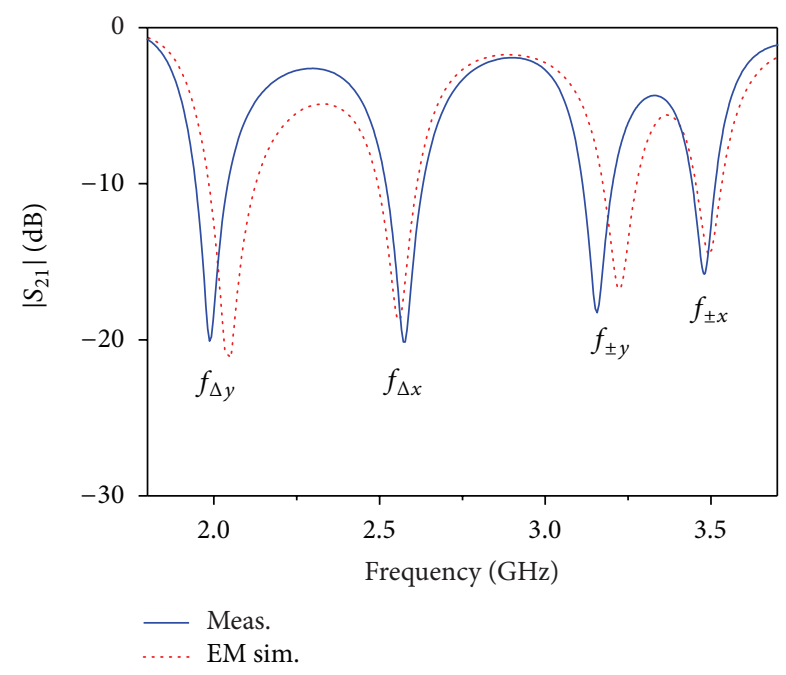

FIGURE 7: Transmission coefficient $S_{21}$ of the sensor for $\Delta x=0.3 \mathrm{~mm}$ and $\Delta y=0.25 \mathrm{~mm}$. From [27], reprinted with permission.

As mentioned before, the position sensing structure was validated by considering several proof-of-concept prototypes with different displacements, by measuring the frequency responses, and by representing the notch magnitude of the different involved resonance frequencies (Figure 8 shows a photograph of the device for the case $\Delta x=\Delta y=0$ ). In order to validate the proposed approach, we considered positive and negative displacement in the $x$-direction (horizontal shift), as well as in the $x=y$-direction (diagonal shift), although here we report only the results corresponding to the horizontal shift.

Figure 9 depicts the dependence of the notch magnitude (simulated and measured) with displacement in the $\pm x$ direction. As expected, for positive displacements, the $S_{R R}$ is activated as is manifested by a clear increase in the notch at $f_{ \pm x}$, whereas the specified $-3 \mathrm{~dB}$ threshold level is not exceeded for negative displacements (indicating that the shift is in the negative direction). The dependence of the notch magnitude for $f_{\Delta x}$ is similar and roughly linear in both directions, with a measured value of approximately $-20 \mathrm{~dB}$ for $\Delta x= \pm 0.3 \mathrm{~mm}$, which is indicative of a significant sensitivity of roughly $65 \mathrm{~dB} / \mathrm{mm}$ (average value). On the other hand, the notch corresponding to $f_{\Delta y}$ is approximately $0 \mathrm{~dB}$, which indicates that the structure is aligned with the $y$ axis (for $\Delta x=0.3 \mathrm{~mm}$ the notch is slightly beyond $-3 \mathrm{~dB}$ because for this sample the $y$-axis position sensing resonators are somewhat misaligned due to fabrication tolerances and underetching). The results of the relative displacement in the diagonal orientation $(\Delta x=\Delta y)$, not shown, indicate that the resonators $\mathrm{SRR}_{ \pm x, \pm y}$ are activated if the displacement is positive.

In comparison to other two-dimensional displacement sensors based on the resonance frequency of split ringloaded transmission lines [17], the proposed sensors based on symmetry properties are more robust against environmental conditions. Indeed, the alignment measurement is immune to (uniform) changes in the properties of the constitutive 


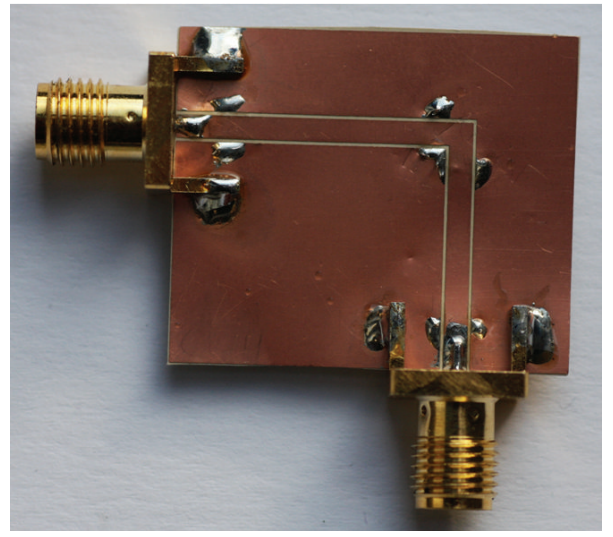

(a)

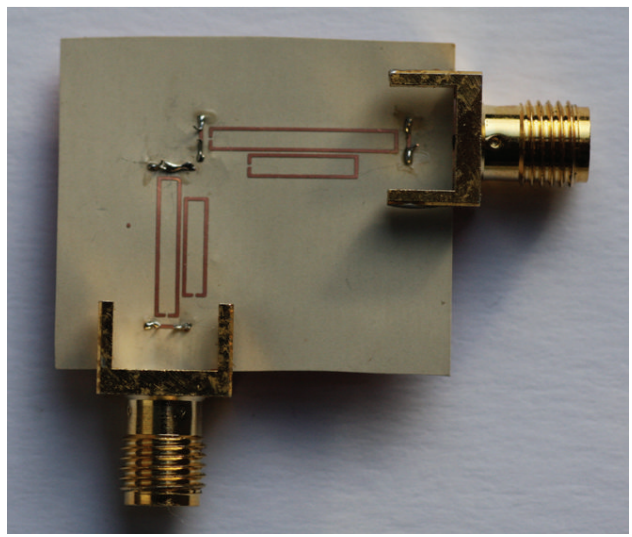

(b)

FIGURE 8: Photograph of the proposed device for the aligned position; (a) top and (b) bottom face. From [27], reprinted with permission.

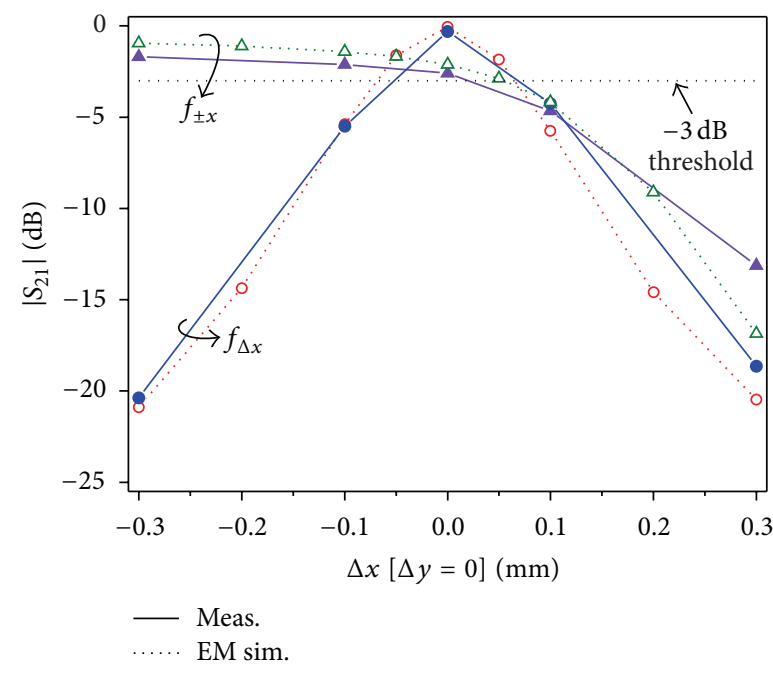

(a)

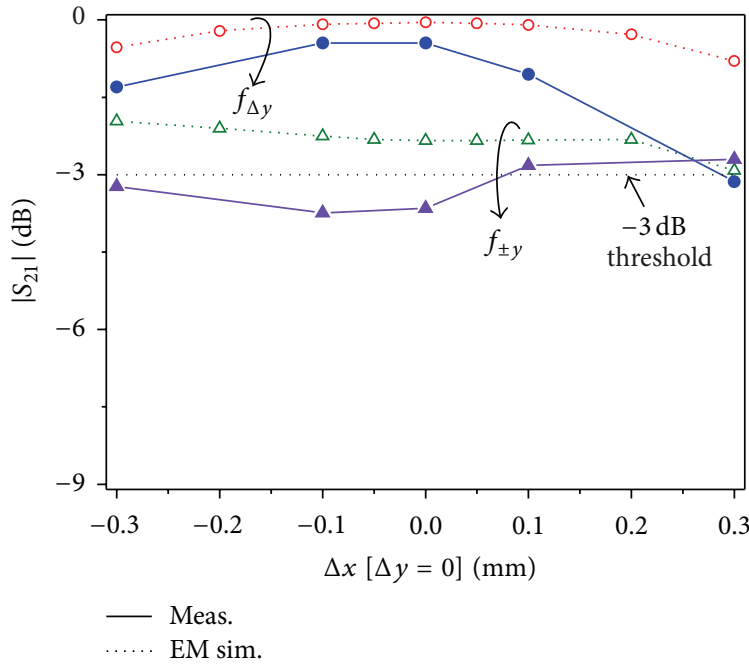

(b)

FIGURE 9: Notch magnitude of the transmission coefficient $S_{21}$ at the indicated frequencies for $x$-oriented displacement; results for (a) $x$ - and (b) $y$-axis position sensing. From [27], reprinted with permission.

materials of the sensors as well as those properties of the surrounding medium.

3.2. Angular Displacement and Velocity Sensors. The angular displacement and velocity sensors reported in this subsection are based on a CPW transmission line loaded with an electricLC (ELC) resonator. The topology of this resonant particle (for a rectangular geometry) is depicted in Figure 10. At the fundamental resonance frequency, the currents in the two loops flow in opposite directions (one is clockwise and the other is counterclockwise), as depicted in that figure. The particle exhibits two symmetry planes, that is, an electric wall and a magnetic wall, as indicated. Hence, according to the sensing principle pointed out in Section 2, it follows that a CPW transmission line loaded with a symmetrically

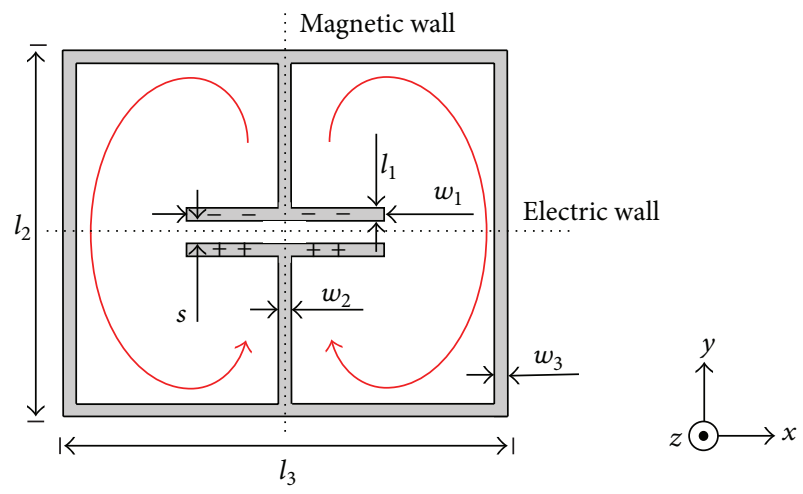

FIGURE 10: Rectangular-shaped electric-LC (ELC) resonator. The electric and magnetic walls at the fundamental resonance, as well as a sketch of the distribution of charges and currents, are indicated. 


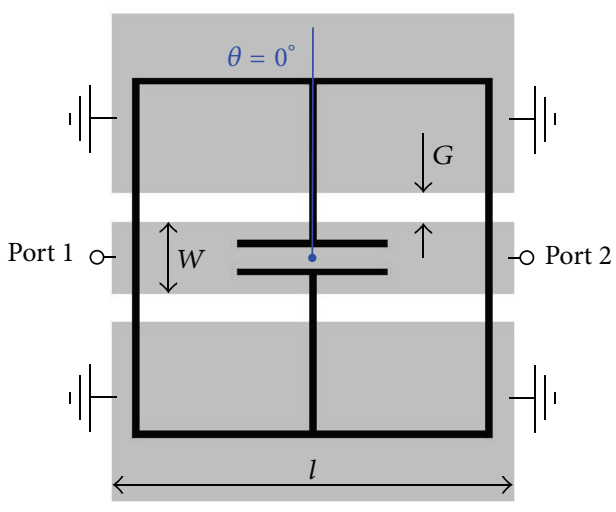

(a)

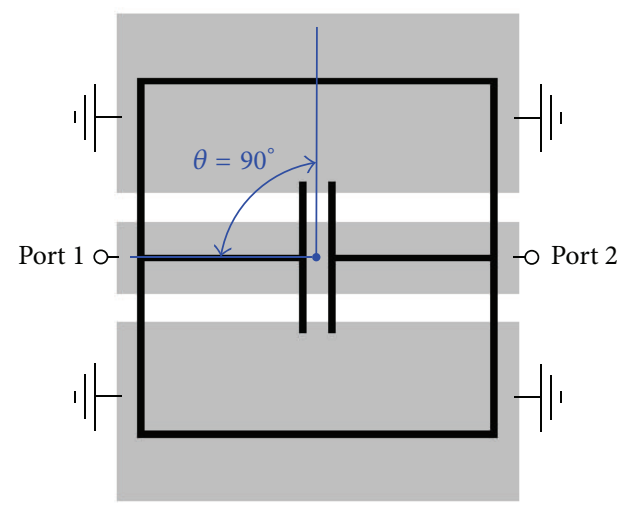

(b)

FIGURE 11: CPW symmetrically loaded with an ELC resonator etched in the back side of the substrate. The orientation between the CPW and the resonator is determined by the rotation angle $\theta$; the line axis is perfectly aligned with the resonator electric wall for $\theta=0^{\circ}$ (a) and with the resonator magnetic wall for $\theta=90^{\circ}$ (b). CPW dimensions are $W=0.78 \mathrm{~mm}$ and $G=2 \mathrm{~mm}$, corresponding to a $50 \Omega$ line. ELC dimensions are $w_{1}=4 \mathrm{~mm}, w_{2}=w_{3}=l_{1}=s=0.2 \mathrm{~mm}$, and $l_{2}=l_{3}=10 \mathrm{~mm}$. The substrate is Rogers $R O 3010$ with $h=1.27 \mathrm{~mm}$ and $\varepsilon_{r}=11.2$.

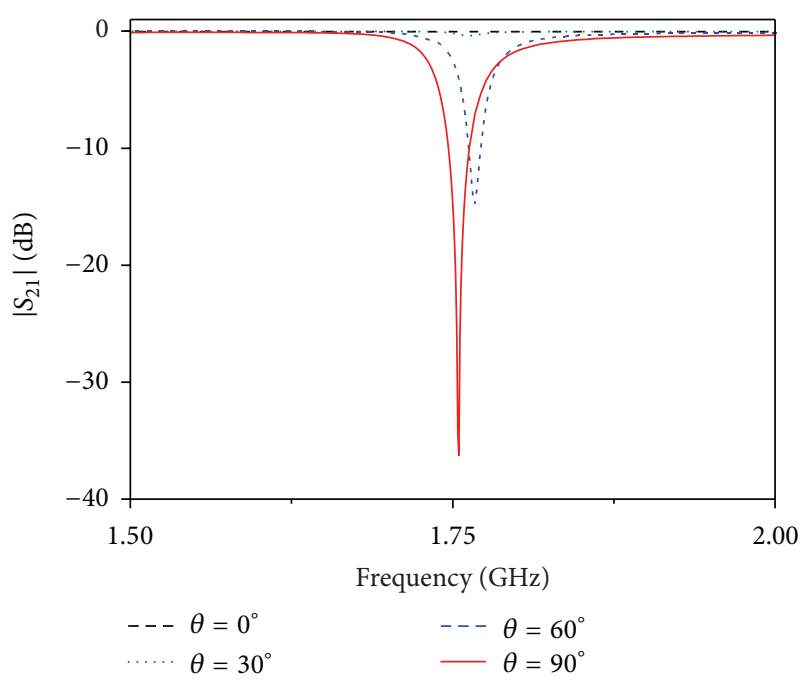

FIGURE 12: Transmission coefficient of the structure of Figure 11 with different resonator rotation angles. The angles $0^{\circ}$ and $90^{\circ}$ correspond to the extreme cases of orthogonally oriented magnetic walls and perfectly aligned magnetic walls, respectively. From [28], reprinted with permission.

etched ELC is transparent if the line axis is aligned with the electric wall of the particle, and a notch is expected to appear if the magnetic wall of the ELC is aligned with the symmetry plane of the line (also a magnetic wall). These two extreme situations are depicted in Figure 11. The frequency response corresponding to these two orientations is shown in Figure 12, where the response for other intermediate angles is also depicted. As expected, the notch magnitude increases with the rotation angle.

A circular geometry for both the ELC resonator and the CPW transmission line is convenient in order to improve sensitivity, dynamic range, and linearity. Thus, we have designed a circular-tapered (nonuniform) CPW transmission line with a characteristic impedance of $50 \Omega$. By designing a circular ELC whose diameter extends beyond the slot regions of the CPW, it is expected that the above sensor parameters are optimized. The ELC has been etched in a different substrate, which has been attached to a rotating cylinder made of Teflon. Rotation can be achieved by means of a step motor. The designed CPW transmission line, ELC resonator, and the experimental setup to measure the rotation angle are shown in Figure 13. After a 3D spatial calibration between the CPW and the resonator by means of manual positioners, the measured notch magnitude and frequency as a function of the rotation are plotted in Figure 14. The results are very similar to those obtained with a suspended substrate and reported in [28]. In comparison to [28], the notch frequency has experienced a slight decrease of $30 \mathrm{MHz}$ due to the Teflon slab, while the change in the notch depth is negligible. These results indicate that the notch magnitude exhibits not only rather robustness, but also less sensitivity than frequencybased sensors to variations or tolerances on the substrate properties (the former may be conditioned to environmental factors). The output dynamic range is $23.7 \mathrm{~dB}$, and the average sensitivity is $0.26 \mathrm{~dB} /{ }^{\circ}$. Therefore, by sensing amplitude levels, remarkable robustness against environmental factors is expected, although noise may degrade the measurements.

The setup of Figure 13 was also used for the measurement of angular velocities. To this end, the CPW transmission line is fed by a harmonic signal (carrier) tuned at the notch frequency of the ELC resonator (actually, the notch frequency slightly depends on the orientation (angle) of the ELC resonator; therefore, the carrier frequency is chosen in the vicinity of the average value). At the output port of the CPW the amplitude of the carrier signal is modulated by the effect of the rotating ELC; that is, the amplitude is maximum each time the electric wall of the ELC is aligned with the symmetry plane of the line (this occurs twice per cycle). Therefore, by capturing the envelope of the modulated signal through an 


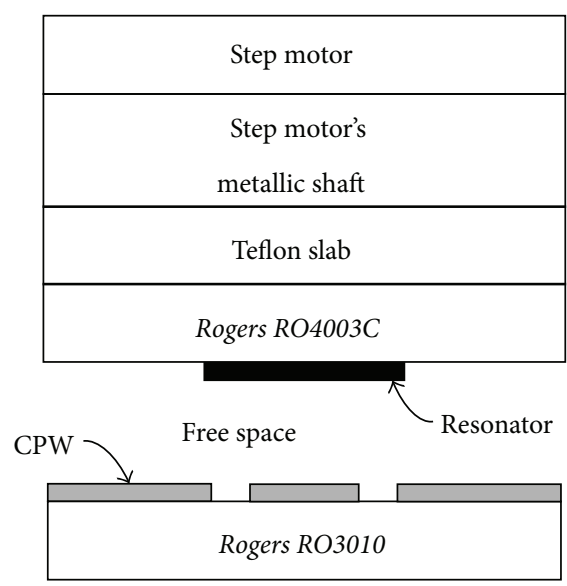

(a)
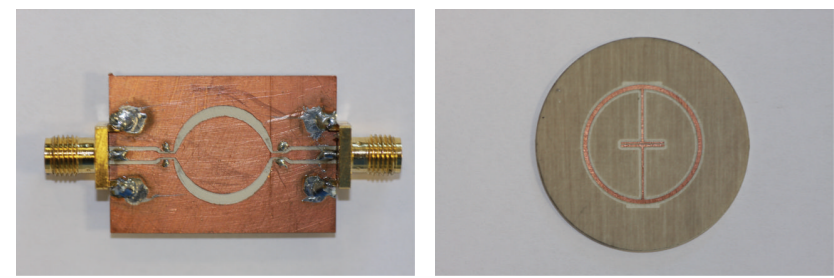

(b)

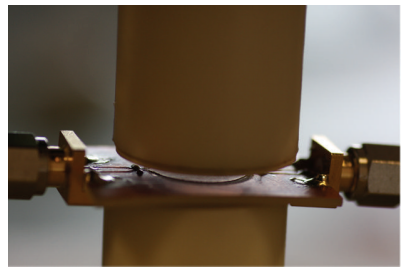

(c)

FIGURE 13: Setup for the angular displacement measurement; (a) layer cross-section, (b) photograph of the fabricated CPW and ELC resonator, and (c) photographs of the experimental setup with positioners and a step motor STM 23Q-3AN. The parameters of the substrates are Rogers $R O 3010, \varepsilon_{r}=11.2, h=1.27 \mathrm{~mm}$, and $\tan \delta=0.0023$; Rogers $R 04003 C, \varepsilon_{r}=3.55, h=0.8128 \mathrm{~mm}$, and $\tan \delta=0.0021 ;$ Teflon, $\varepsilon_{r}=2.08, h=3.5 \mathrm{~mm}$, and $\tan \delta=0.0004$; in-between air layer, $h=1.27 \mathrm{~mm}, \varepsilon_{r}=1$, and $\tan \delta=0$. ELC dimensions are mean radius $r_{0}=8.05 \mathrm{~mm}, l_{1}=0.2 \mathrm{~mm}$ and $w_{1}=6 \mathrm{~mm}, w_{2}=s=0.2 \mathrm{~mm}$, and $w_{3}=0.5 \mathrm{~mm}$. From [33], reprinted with permission.

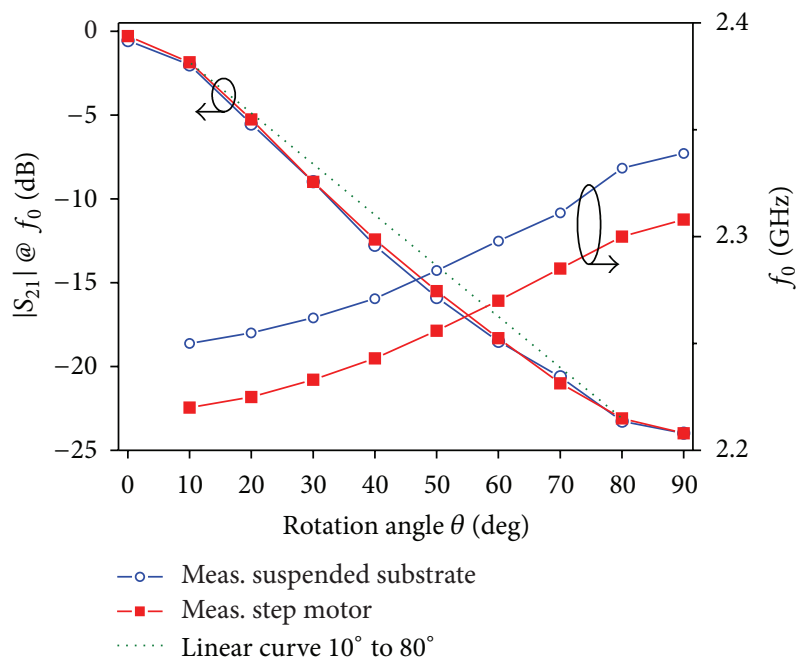

FIgURE 14: Transfer function of the measured notch magnitude at the notch frequency in the transmission coefficient versus the rotation angle. The experimental setup of Figure 13 was used. From [33], reprinted with permission.

envelope detector, the distance between adjacent peaks gives the rotation speed. In order to avoid unwanted reflections caused by the envelope detector, a circulator is cascaded between the output port of the CPW transmission line and the input of the envelope detector, as depicted in Figure 15(a). The typical waveform of the envelope signal obtained through an oscilloscope, for a rotation speed of 50 cycles per second, is depicted in Figure 15(b). The pronounced peaks allow for the measurement of angular velocities with high accuracy (errors less than $1 \%$ have been obtained). Since the carrier frequency is very high, it is obvious that very high angular velocities can be measured with this approach. The microstrip version of this angular velocity sensor was reported in [34].

As stressed before, the main advantage of the proposed symmetry-based sensors is their inherent immunity to changing ambient conditions. By contrast, angular sensors based on the shift of resonance frequency may be affected by environmental factors [22]. In addition, as compared to spatial sensors with mechanical friction (e.g., rotary potentiometers [42]), the proposed sensors are contactless and therefore do not suffer from aging effects caused by friction. It should be noted that not only may the performance of microwave sensors be subjected to changing ambient conditions, but that of sensors is based on other sensing principles (e.g., optical sensors [43]).

\section{Conclusion}

In conclusion, a novel type of microwave sensors, recently proposed by the authors and based on the symmetry properties of resonator-loaded transmission lines, has been 


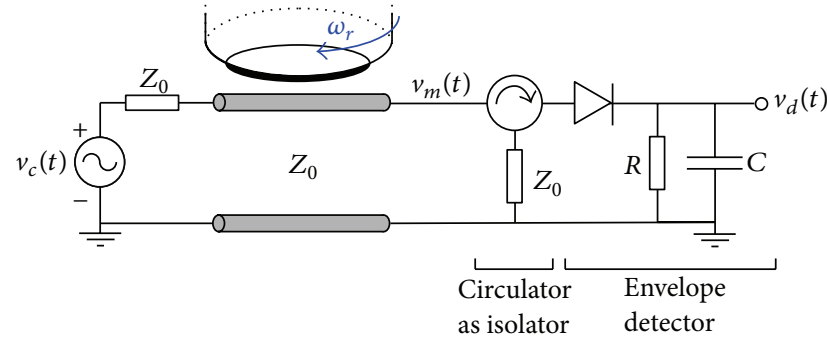

(a)

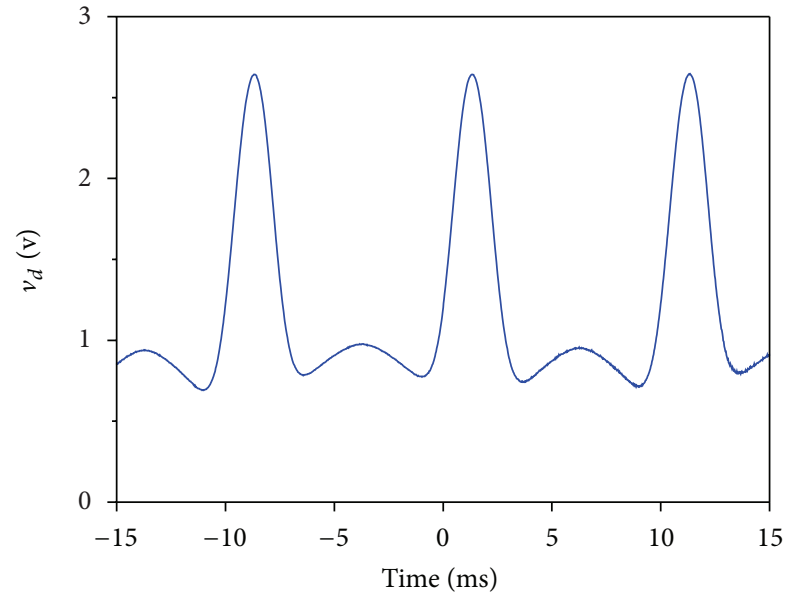

(b)

FIGURE 15: Schematic of the proposed system for measuring angular velocities in time domain, including the sensing line, circulator and envelope detector (a), and output waveform for a rotating speed of 50 cycles per second (b). From [33], reprinted with permission.

reviewed. The sensing principle is based on the disruption of symmetry caused by a certain variable, such as position, angle, or permittivity of a dielectric load. The asymmetry produces a notch in the transmission coefficient, whose depth depends on the level of asymmetry (which in turn determines the net coupling between the line and the resonator). Therefore, the proposed structures are useful for sensing purposes. Two different examples of sensors have been discussed: a linear position/alignment sensor and an angular alignment/displacement/velocity sensor. The reported results of sensor characterization reveal that the sensors exhibit a reasonable sensitivity, linearity, and dynamic range. Moreover, the proposed sensors are robust against changes in environmental conditions and particularly in alignment measurements since geometrical symmetry is not influenced by such changes.

\section{Conflict of Interests}

The authors declare that there is no conflict of interests regarding the publication of this paper.

\section{Acknowledgments}

This work has been supported by MINECO, Spain (Projects TEC2010-17512, CSD2008-00066, TEC2011-13615-E, and TEC2013-40600-R), with FEDER Funds, and by AGAURGeneralitat de Catalunya, through Project 2014SGR-157. Jordi Naqui is also in debt to MECD (Spain) for supporting his work through the FPU Grant AP2010-0431. Ferran Martín is in debt to ICREA for supporting his work through an ICREA Academia Award.

\section{References}

[1] F. Martín, F. Falcone, J. Bonache, R. Marqués, and M. Sorolla, "Split ring resonator-based left-handed coplanar waveguide," Applied Physics Letters, vol. 83, no. 22, pp. 4652-4654, 2003.
[2] F. Falcone, T. Lopetegi, J. D. Baena, R. Marqués, F. Martín, and M. Sorolla, "Effective negative- $\varepsilon$ stopband microstrip lines based on complementary split ring resonators," IEEE Microwave and Wireless Components Letters, vol. 14, no. 6, pp. 280-282, 2004.

[3] L. J. Roglá, J. Carbonell, and V. E. Boria, "Study of equivalent circuits for open-ring and split-ring resonators in coplanar waveguide technology," IET Microwaves, Antennas and Propagation, vol. 1, no. 1, pp. 170-176, 2007.

[4] S. N. Khan, Q. L. Zhang, and S. He, "Left handed microstrip transmission line loaded with combination of split ring resonator and complementary-SRR," Journal of Electromagnetic Waves and Applications, vol. 22, no. 13, pp. 1857-1863, 2008.

[5] M. Durán-Sindreu, A. Vélez, F. Aznar, G. Sisó, J. Bonache, and F. Martín, "Applications of open split ring resonators and open complementary split ring resonators to the synthesis of artificial transmission lines and microwave passive components," IEEE Transactions on Microwave Theory and Techniques, vol. 57, no. 12, pp. 3395-3403, 2009.

[6] I. A. I. Al-Naib and M. Koch, "Coplanar waveguides incorporating SRRS or CSRRS," Progress in Electromagnetics Research $B$, vol. 23, pp. 343-355, 2010.

[7] G. Disken, F. Pala, E. Demir, H. D. Korucu, and E. Ekmekci, "Electromagnetic behavior of SRR loaded microstrip transmission lines: investigation for different SRR types and array topologies," in Proceedings of the Progress in Electromagnetics Research Symposium (PIERS '13), pp. 1796-1799, Stockholm, Sweden, August 2013.

[8] F. Falcone, J. Illescas, E. Jarauta, A. Estevez, and J. A. Marcotegui, "Analysis of stripline configurations loaded with complementary split ring resonators," Microwave and Optical Technology Letters, vol. 55, no. 6, pp. 1250-1254, 2013.

[9] G. V. Eleftheriades and K. G. Balmain, Eds., Negative-Refraction Metamaterials: Fundamental Principles and Applications, John Wiley \& Sons, New York, NY, USA, 2005.

[10] C. Caloz and T. Itoh, Electromagnetic Metamaterials: Transmission Line Theory and Microwave Applications, John Wiley \& Sons, New York, NY, USA, 2006. 
[11] R. Marqués, F. Martín, and M. Sorolla, Metamaterials with Negative Parameters: Theory, Design and Microwave Applications, John Wiley \& Sons, New York, NY, USA, 2008.

[12] L. Solymar and E. Shamonina, Waves in Metamaterials, Oxford University Press, Oxford, UK, 2009.

[13] F. Martín, Artificial Transmission Lines for RF and Microwave Applications, John Wiley \& Sons, New York, NY, USA, 2015.

[14] J. B. Pendry, A. J. Holden, D. J. Robbins, and W. J. Stewart, "Magnetism from conductors and enhanced nonlinear phenomena," IEEE Transactions on Microwave Theory and Techniques, vol. 47, no. 11, pp. 2075-2084, 1999.

[15] V. G. Veselago, "The electrodynamics of substances with simultaneously negative values of $\varepsilon$ and $\mu$," Soviet Physics Uspekhi, vol. 10, no. 4, pp. 509-514, 1968.

[16] D. R. Smith, W. J. Padilla, D. C. Vier, S. C. Nemat-Nasser, and S. Schultz, "Composite medium with simultaneously negative permeability and permittivity," Physical Review Letters, vol. 84, no. 18, pp. 4184-4187, 2000.

[17] C. Mandel, B. Kubina, M. Schüßler, and R. Jakoby, "Passive chipless wireless sensor for two-dimensional displacement measurement," in Proceedings of the 41st EuropeanMicrowave Conference (EuMC '11), pp. 79-82, Manchester, UK, October 2011.

[18] M. Puentes, C. Weiss, M. Schüßler, and R. Jakoby, "Sensor array based on split ring resonators for analysis of organic tissues," in Proceedings of the IEEE MTT-S International Microwave Symposium (IMS '11), Baltimore, Md, USA, June 2011.

[19] W. Withayachumnankul, K. Jaruwongrungsee, A. Tuantranont, C. Fumeaux, and D. Abbott, "Metamaterial-based microfluidic sensor for dielectric characterization," Sensors and Actuators A: Physical, vol. 189, pp. 233-237, 2013.

[20] A. Ebrahimi, W. Withayachumnankul, S. Al-Sarawi, and D. Abbott, "High-sensitivity metamaterial-inspired sensor for microfluidic dielectric characterization," IEEE Sensors Journal, vol. 14, no. 5, pp. 1345-1351, 2014.

[21] A. A. Abduljabar, D. J. Rowe, A. Porch, and D. A. Barrow, "Novel microwave microfluidic sensor using a microstrip splitring resonator," IEEE Transactions on Microwave Theory and Techniques, vol. 62, no. 3, pp. 679-688, 2014.

[22] A. Ebrahimi, W. Withayachumnankul, S. Al-Sarawi, and D. Abbott, "Metamaterial-inspired rotation sensor with wide dynamic range," IEEE Sensors Journal, vol. 14, no. 8, pp. 26092614, 2014.

[23] T. Chen, S. Li, and H. Sun, "Metamaterials application in sensing," Sensors, vol. 12, no. 3, pp. 2742-2765, 2012.

[24] E. Ekmekci and G. Turhan-Sayan, "Multi-functional metamaterial sensor based on a broad-side coupled SRR topology with a multi-layer substrate," Applied Physics A, vol. 110, no. 1, pp. 189197, 2013.

[25] J. Naqui, M. Durán-Sindreu, and F. Martín, "Novel sensors based on the symmetry properties of split ring resonators (SRRs)," Sensors, vol. 11, no. 8, pp. 7545-7553, 2011.

[26] J. Naqui, M. Durán-Sindreu, and F. Martín, “On the symmetry properties of coplanar waveguides loaded with symmetric resonators: analysis and potential applications," in Proceedings of the IEEE MTT-S International Microwave Symposium Digest (IMS '12), pp. 1-3, IEEE, Montreal, Canada, June 2012.

[27] J. Naqui, M. Durán-Sindreu, and F. Martín, "Alignment and position sensors based on split ring resonators," Sensors, vol. 12, no. 9, pp. 11790-11797, 2012.
[28] J. Naqui, M. Durán-Sindreu, and F. Martín, “Transmission lines loaded with bisymmetric resonators and applications," in Proceedings of the IEEE MTT-S International Microwave Symposium Digest (IMS '13), pp. 1-3, Seattle, Wash, USA, June 2013.

[29] J. Naqui and F. Martín, "Mechanically reconfigurable microstrip lines loaded with stepped impedance resonators and potential applications," International Journal of Antennas and Propagation, vol. 2014, Article ID 346838, 8 pages, 2014.

[30] A. Karami-Horestani, C. Fumeaux, S. F. Al-Sarawi, and D. Abbott, "Displacement sensor based on diamond-shaped tapered split ring resonator," IEEE Sensors Journal, vol. 13, no. 4, pp. 1153-1160, 2013.

[31] A. K. Horestani, D. Abbott, and C. Fumeaux, "Rotation sensor based on horn-shaped split ring resonator," IEEE Sensors Journal, vol. 13, no. 8, pp. 3014-3015, 2013.

[32] A. K. Horestani, J. Naqui, D. Abbott, C. Fumeaux, and F. Martín, "Two-dimensional displacement and alignment sensor based on reflection coefficients of open microstrip lines loaded with split ring resonators," IET Electronics Letters, vol. 50, no. 8, pp. 620-622, 2014.

[33] J. Naqui and F. Martín, "Transmission lines loaded with bisymmetric resonators and their application to angular displacement and velocity sensors," IEEE Transactions on Microwave Theory and Techniques, vol. 61, no. 12, pp. 4700-4713, 2013.

[34] J. Naqui and F. Martín, "Angular displacement and velocity sensors based on electric-LC (ELC) loaded microstrip lines," IEEE Sensors Journal, vol. 14, no. 4, pp. 939-940, 2014.

[35] A. K. Horestani, J. Naqui, Z. Shaterian, D. Abbott, C. Fumeaux, and F. Martín, "Two-dimensional alignment and displacement sensor based on movable broadside-coupled split ring resonators," Sensors and Actuators A: Physical, vol. 210, pp. 18-24, 2014.

[36] J. Naqui, C. Damm, A. Wiens, R. Jakoby, L. Su, and F. Martín, "Transmission lines loaded with pairs of magnetically coupled stepped impedance resonators (SIRs): modeling and application to microwave sensors," in Proceedings of the IEEE/MTT-S International Microwave Symposium (IMS '14), pp. 1-4, Tampa, Fla, USA, June 2014.

[37] M. Schueler, C. Mandel, M. Puentes, and R. Jakoby, "Metamaterial inspired microwave sensors," IEEE Microwave Magazine, vol. 13, no. 2, pp. 57-68, 2012.

[38] J. J. Yang, M. Huang, H. Tang, J. Zeng, and L. Dong, "Metamaterial sensors," International Journal of Antennas and Propagation, vol. 2013, Article ID 637270, 16 pages, 2013.

[39] M. Huang, J. Yang, J. Wang, and J. Peng, "Microwave sensor for measuring the properties of a liquid drop," Measurement Science and Technology, vol. 18, no. 7, pp. 1934-1938, 2007.

[40] M. Makimoto and S. Yamashita, "Compact bandpass filters using stepped impedance resonators," Proc IEEE, vol. 67, no. 1, pp. 16-19, 1977.

[41] D. Schurig, J. J. Mock, and D. R. Smith, "Electric-field-coupled resonators for negative permittivity metamaterials," Applied Physics Letters, vol. 88, no. 4, Article ID 041109, 2006.

[42] J. Fraden, Handbook of Modern Sensors: Physics, Design, and Applications, Springer, New York, NY, USA, 3rd edition, 2004.

[43] J. G. Webster, The Measurement Instrumentation and Sensors Handbook, CRC Press, Boca Raton, Fla, USA, 1999. 

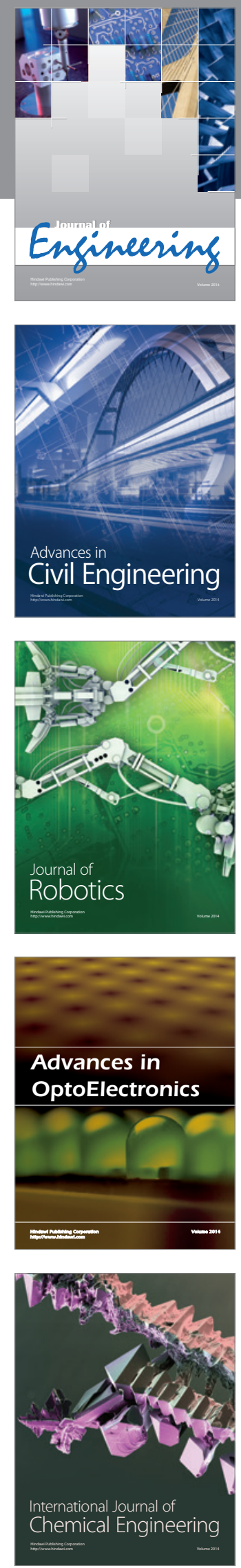

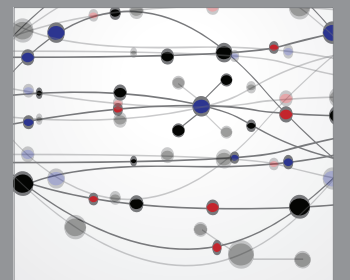

The Scientific World Journal
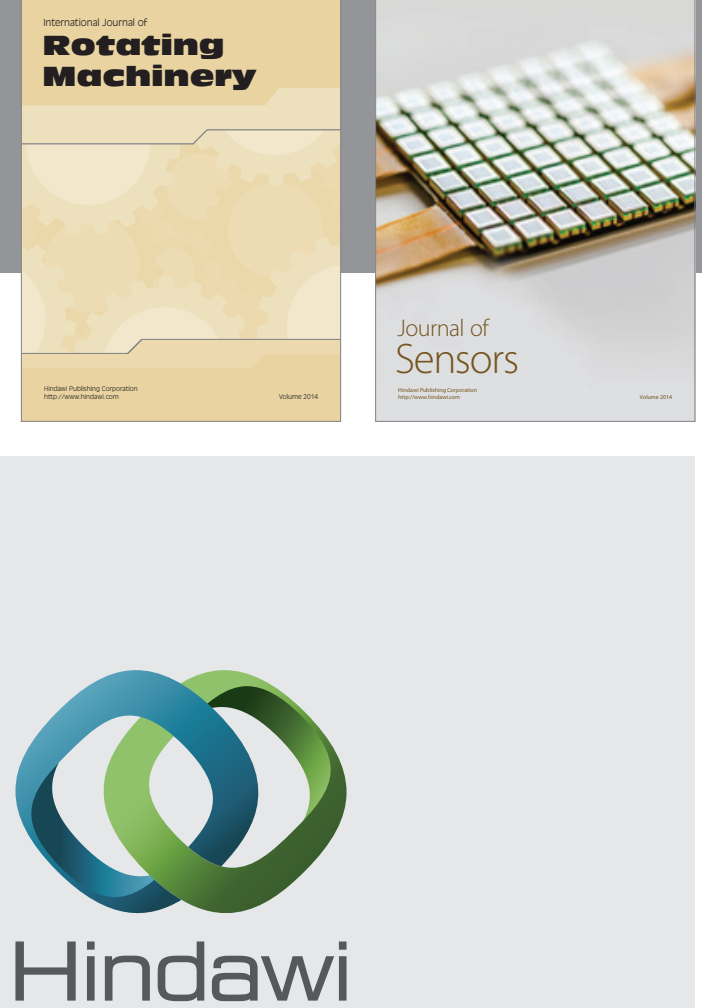

Submit your manuscripts at http://www.hindawi.com
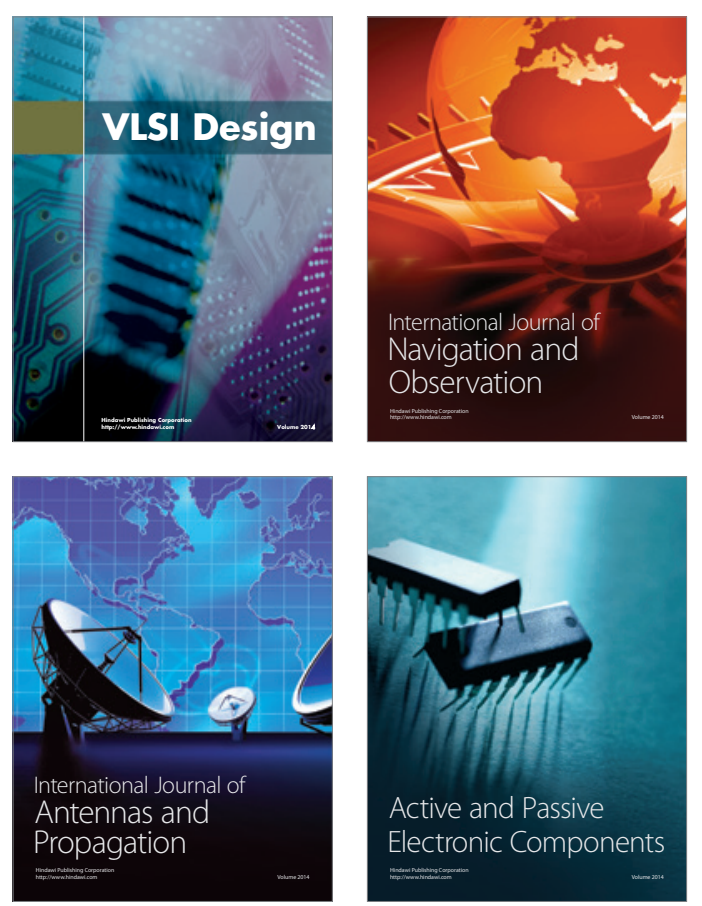
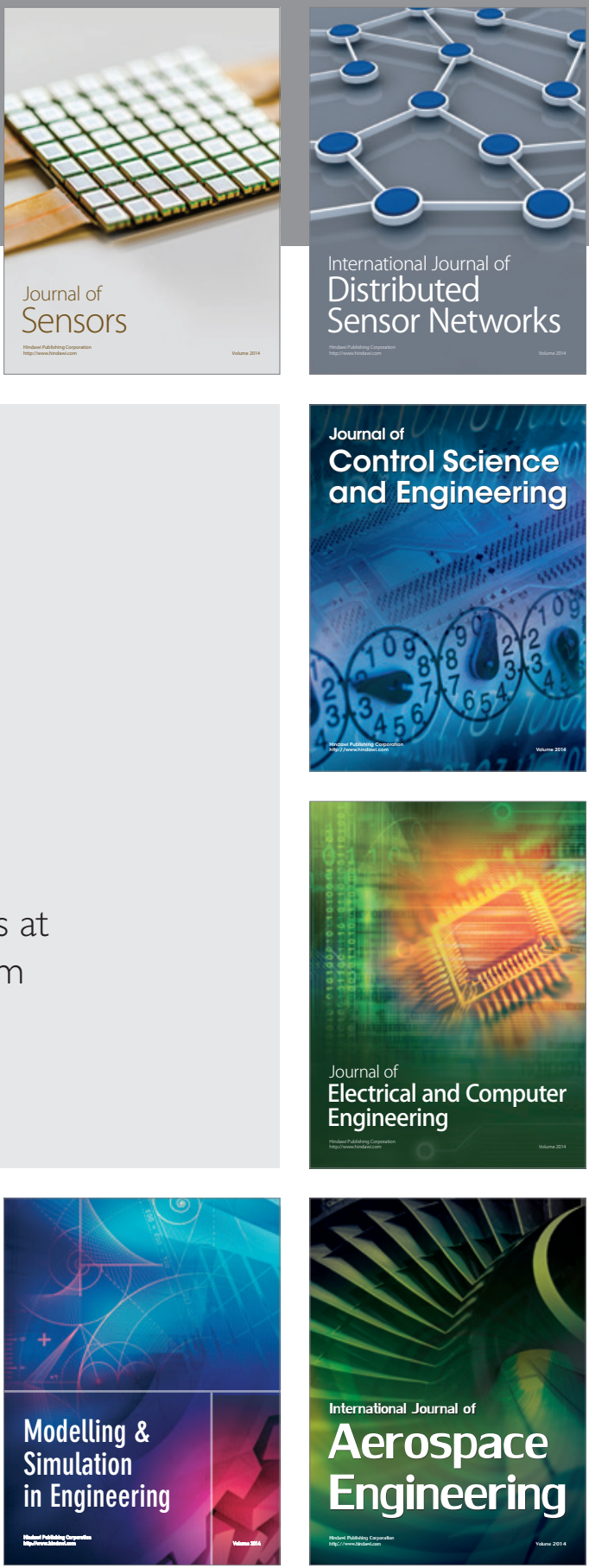

Journal of

Control Science

and Engineering
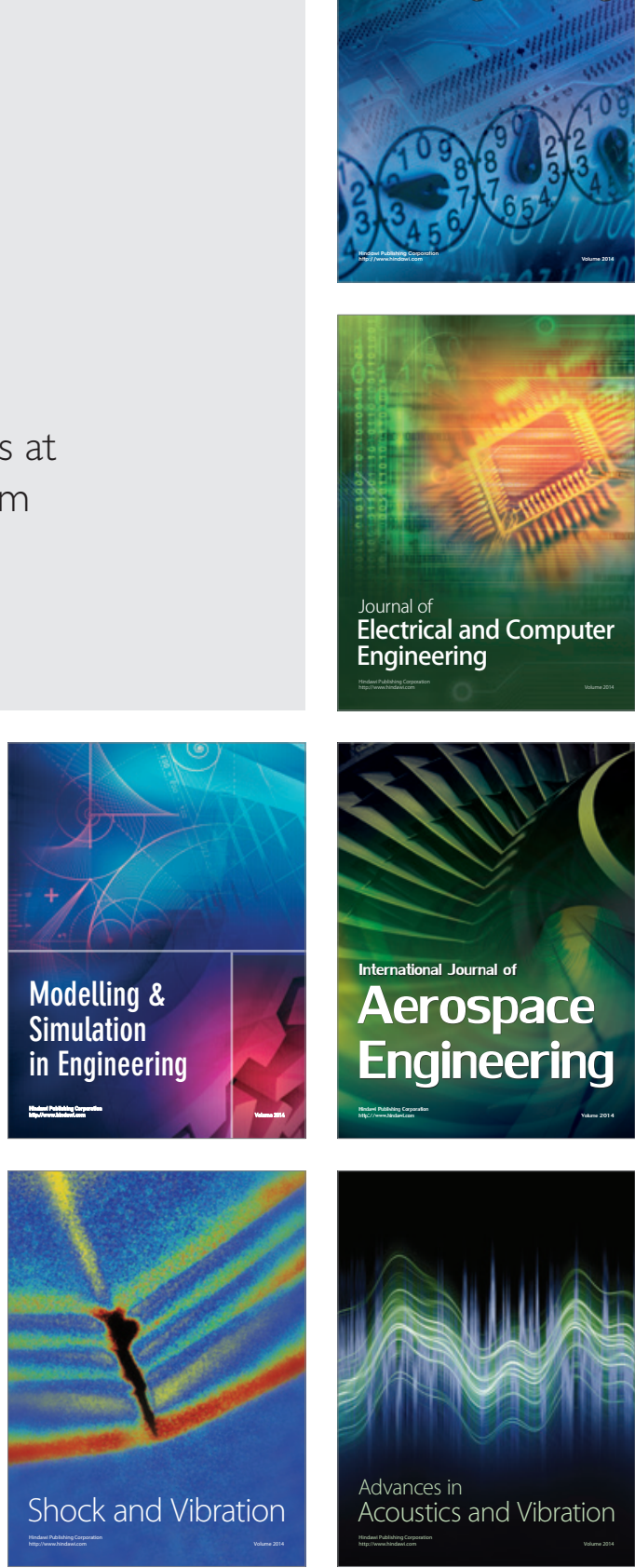